\title{
On Local Characterization of Wave Front Sets in Terms of Boundary Values of Holomorphic Functions
}

\author{
By
}

\author{
Kimimasa NisHIWADA*
}

\section{§1. Introduction}

Let $f$ be a distribution defined in an open set $X$ in $\mathbb{R}^{n}$. $L$. Hörmander [4] introduced the notion of the analytic wave front set $W F_{A}(f)$ of $f$ as a subset of the cotangent space $T^{*}(X) \backslash 0$ whose projection to $X$ coincides with the analytic singular support of $f$. His definition relies on the use of the Fourier transform of $f$. In this paper we present an alternative definition of $W F_{A}(f)$ in terms of boundary values of holomorphic functions which we now shortly describe.

Let $\Omega$ be an open subset in $C^{n}$. Then we shall denote by $\mathcal{O}(\Omega)$ the space of holomorphic functions in $\Omega$.

Definition 1. 1. Let $U$ be an open subset of $X, \tilde{U}$ a complex neighborhood of $U$ such that $\tilde{U} \cap \boldsymbol{R}^{n}=U$ and $\Gamma$ an open convex cone in $\mathbb{R}^{n}$ with vertex at the origin. We say that a function $f \in$ $\mathcal{O}(\tilde{U} \cap T(\Gamma))$ admits the boundary value $f(x+i \Gamma 0)$ in $\mathscr{D}^{\prime}(U)$ if the limit of $f(x+i y)$ exists in $\mathscr{D}^{\prime}(U)$ as $\Gamma^{\prime} \ni y \rightarrow 0$ for every proper subcone $\Gamma^{\prime} \Subset \Gamma$. Here we have put $T(\Gamma)=\mathbb{R}^{n}+i \Gamma$.

In this article the boundary values of holomorphic functions are always considered in the distribution sense defined above. We can now state our main result.

Received November 2, 1976.

* Research Institute for Mathematical Sciences, Kyoto University, Kyoto 606, Japan. 
Theorem 1.2. Let $f \in \mathscr{D}^{\prime}(X)$ and $\left(x_{0}, \xi_{0}\right) \in T^{*}(X) \backslash 0$. Then $\left(x_{0}, \xi_{0}\right) \notin W F_{A}(f)$ if and only if there exists a finite family $\left\{\Gamma_{a}\right\}$ of open convex cones in $\mathbb{R}^{n}$, a complex neighborhood $\tilde{U}$ of $x_{0}$ and a decomposition of $f$

$$
f(x)=\sum_{\alpha} f_{\alpha}\left(x+i \Gamma_{\alpha} 0\right) \text { near } x_{0}
$$

with such $f_{\alpha} \in \mathcal{O}\left(\tilde{U} \cap T\left(\Gamma_{\alpha}\right)\right)$ that $f_{\alpha}$ is analytic close to $x_{0}$ for every $\alpha$ satisfying $\Gamma_{\alpha} \subset\left\{y ;<y, \hat{\varsigma}_{0}>\geqq 0\right\}$.

It was M. Sato [7] that first introduced the concept of hyperfunction defined a priori as a sum of boundary values of holomorphic functions. On the other hand, the theory of distribution boundary value of holomorphic function is developed by A. Martineau [5].

If in (1.1) no growth condition on each $f_{\alpha}(x+i \Gamma 0)$ is posed, this leads to a definition of microanalyticity for hyperfunction and then to the theory of sheaf $\mathscr{C}$ (see [8]). The microlocal study in the distribution boundary value case was investigated in BrosIagornitzer [1], however the relation to the analytic wave front set was not discussed there.

In $\S 2$ of this paper, we give a simpler proof to one of the fundamental results in [5]. As well as the result, some part of its proof will be useful in the proofs of Theorem 1.2 and other results in $\S 3$.

A summary of this paper was given in [6] with an application to the theory of partial differential equations.

The author would like to express his sincere gratitude to Professor S. Matsuura and Dr. N. Iwasaki for valuable advice and kind interest.

\section{§2. Boundary Values of Holomorphic Functions}

In this section we shall examine Martineau's criterion for the existence of distribution boundary value from a new viewpoint. First we need the following lemma whose implication $(a) \Rightarrow(b)$ is known (see [3]). 
Lemma 2.1. Let $U$ be an open set in $X$ and $\tilde{U}$ a complex neighborhood of $U$ such that $\tilde{U} \cap \boldsymbol{R}^{n}=U$. Then the following statements are equivalent for a function $u \in C_{0}^{1}(U)$.

(a) $u \in C_{0}^{\infty}(U)$

(b) There exists an extension $\tilde{u}(x+i y) \in C_{0}^{1}(\tilde{U})$ of $u$ such that

$$
\sup _{x \in U}|\bar{\partial} \tilde{u}(x+i y)| \leqq C_{N}|y|^{N}, N=1,2, \ldots,
$$

where $\bar{\partial}$ is the Cauchy-Riemann operator.

Proof. First assume that $u \in C_{0}^{\infty}(U)$. Then one can construct $\tilde{u}(x+i y)$ as follows.

$$
\tilde{u}(x+i y)=\sum_{\alpha} u^{(\alpha)}(x)(i y)^{\alpha} \chi\left(b_{|\alpha|} y\right) / \alpha !
$$

where the function $\chi \in C_{0}^{\infty}\left(\mathbb{R}^{n}\right)$ is chosen so that $\chi(y)=1$ if $|y| \leqq \frac{1}{2}$ and $\chi(y)=0$ if $|y| \geqq 1$ and the positive increasing sequence $\left\{b_{n}\right\}_{n=0}^{\infty}$ so that $u^{(\alpha)}(x)(i y)^{\alpha} \chi\left(b_{|\alpha|} y\right)$ are bounded in $C^{j}(\tilde{U})$ for every $j$. It follows then that $\tilde{u} \in C_{0}^{\infty}$ and that the functions

$$
\begin{aligned}
\frac{\partial \tilde{u}}{\partial \bar{z}_{j}}(x+i y) & =\sum_{\alpha}\left\{u^{\left(\alpha+1_{j}\right)}(x)(i y)^{\alpha}\left(\chi\left(b_{|\alpha|} y\right)-\chi\left(b_{|\alpha|+1} y\right)\right)\right. \\
& \left.+u^{(\alpha)}(x)(i y)^{\alpha} b_{|\alpha|} \chi_{j}^{\prime}\left(b_{|\alpha|} y\right)\right\} / 2 \alpha !
\end{aligned}
$$

are bounded by $C_{N}|y|^{N}, N=1,2, \ldots$

Conversely we assume the existence of $\tilde{u} \in C_{0}^{1}(\tilde{U})$ satisfying(2.1). In order to express $u$ as a function of $\bar{\partial} \tilde{u}$, we use the plane wave expansion formula of Dirac function, that is

$$
\delta(x)=-\frac{(n-1) !}{(-2 \pi i)^{n}} \int_{|\xi|=1} \frac{\omega(\xi)}{(\langle x, \xi\rangle+i 0)^{n}}
$$

where $\omega(\xi)=\sum(-1)^{j-1} \xi_{j} d \xi_{1} \wedge \ldots \wedge d \xi_{j-1} \wedge d \xi_{j+1} \ldots \wedge d \xi_{n}$. (2.2) means for any $\varphi \in C_{0}^{\infty}(U)$ and its extension $\tilde{\varphi} \in C_{0}^{\infty}(\tilde{U})$ satisfying (2.1)

$$
\begin{aligned}
\langle\delta(x), \varphi(x)\rangle & =\frac{(n-1) !}{(-2 \pi i)^{n}} \int_{|\xi|=1} \int \frac{\varphi(x) d x}{(\langle x, \xi\rangle+i 0)^{n}} \omega(\xi) \\
& =\frac{(n-1) !}{(-2 \pi i)^{n}} \int_{B} \frac{\bar{\partial} \tilde{\varphi}(z) \wedge d z \wedge \omega(\xi)}{\langle z, \xi\rangle^{n}} .
\end{aligned}
$$

Here the last equality follows from the application of Stokes formula 
if we set

$$
B=\left\{z=x+i t y_{\xi} ; \xi \in S^{n-1}, x \in \boldsymbol{R}^{n}, t>0\right\}
$$

with a continuous vector field $y_{\xi}$ on $S^{n-1}$ admitting $\left\langle y_{\xi}, \xi\right\rangle>0$ and give the orientation by $d t \wedge d x \wedge \omega(\xi)<0$. In view of (2.1) the integral (2.3) is absolutely convergent. Taking suitable approximations $\varphi_{n} \rightarrow u$ and $\tilde{\varphi}_{n} \rightarrow \tilde{u}$ in $C^{1},{ }^{1)}$ we have thus

$$
\begin{aligned}
u(x) & =\left\langle u\left(x-x^{\prime}\right), \delta\left(x^{\prime}\right)\right\rangle \\
& =\frac{-(n-1) !}{(-2 \pi i)^{n}} \int_{B} \frac{\bar{\partial} \tilde{u}\left(x-z^{\prime}\right) \wedge d z^{\prime} \wedge \omega(\xi)}{\left\langle z^{\prime}, \xi\right\rangle^{n}} \\
& =\frac{-(n-1) !}{(-2 \pi i)^{n}} \int_{B^{\prime}} \frac{\bar{\partial} \tilde{u}\left(z^{\prime}\right) \wedge d z^{\prime} \wedge \omega(\xi)}{\left\langle z^{\prime}-x, \xi\right\rangle^{n}}
\end{aligned}
$$

for suitable $B^{\prime}$. This shows $u \in C_{0}^{\infty}$ and completes the proof.

We shall now prove a fundamental result of Martineau [5].

Theorem 2.2. Let $U, \tilde{U}$ and $\Gamma$ be as in Definition 1. 1. Then the following conditions a re equivalent for a function $f \in \mathcal{O}(\tilde{U} \cap T(\Gamma))$. For any $\omega \Subset U$ and any convex cone $\Gamma^{\prime} \Subset \Gamma$;

(a) the limit of $f(x+i y)$ as $\Gamma^{\prime} \ni y \rightarrow 0$ exists in $\mathscr{D}^{\prime}(\omega)$ that is, $f$ admits the distribution boundary value $f(x+i \Gamma 0)$.

(b) the functions $\{f(x+i y)\}$ of $x \in \omega$ with small $y \in \Gamma^{\prime}$ form a bounded set in $\mathscr{D}^{\prime}(\omega)$.

(c) there exist positive numbers $k, \delta$ and $C$ such that

$$
\left|\iint f(x+i y) \varphi(x, y) d x d y\right| \leqq C \sup _{|\alpha| \leqq k}\left|D_{x}^{\alpha} \varphi\right|
$$

for all $\varphi \in C_{0}^{\infty}\left(\left(\omega+i \Gamma^{\prime}\right) \cap\{|y| \leqq \delta\}\right)$.

(d) there exist positive numbers $C$ and $M$ such that

$$
\sup _{x \in \omega}|f(x+i y)| \leqq C|y|^{-M}
$$

for small $y \in \Gamma^{\prime}$.

Remark. If $f$ satisfies (2.5), then it follows from Cauchy's integral formula that with another constant $C$

1) We may define $\tilde{\varphi}_{n}=\tilde{u} *_{x} \rho_{1 / n}$ employing the usual mollifier. 


$$
\sup _{x \in \omega}\left|D_{x}^{\alpha} f(x+i y)\right| \leqq C(C|\alpha|)^{|\alpha|}|y|^{-M-|\alpha|}
$$

for small $y \in \Gamma^{\prime}$.

Proof. The implication (a) $\Rightarrow(\mathrm{b})$ is obvious. To prove (b) $\Rightarrow(\mathrm{c})$, we note that the functions $f(x+i y)$ with small $y \in \Gamma^{\prime}$ are equicontinuous on $C_{0}^{\infty}(\omega)$ since they are bounded. This implies with small $\delta>0$

$$
\begin{aligned}
& \left|\int f(x+i y) \varphi(x, y) d x\right| \leqq C \sup _{x \in \omega,|\alpha| \leqq k}\left|D_{x}^{\alpha} \varphi(x, y)\right|, \\
& \varphi \in C_{0}^{\infty}\left(\left(\omega^{\prime}+i \Gamma^{\prime}\right) \cap\{|y| \leqq \delta\}\right)
\end{aligned}
$$

where $C$ and $k$ are independent of $y \in \Gamma^{\prime}$. Thus, integrating (2.7) in $y$ variables, we obtain (2.4).

Next assume (c) is valid and choose $\omega^{\prime}$ and $\Gamma^{\prime \prime}$ so as to be $\omega+$ $i \Gamma^{\prime} \Subset \omega^{\prime}+i \Gamma^{\prime \prime} \Subset U+i \Gamma$. One can find small $\varepsilon>0$ so that for any $z=$ $x+i y \in \omega+i \Gamma^{\prime}, \operatorname{dist}\left(x, \partial \omega^{\prime}\right)>\varepsilon$ and $\operatorname{dist}\left(y, \partial \Gamma^{\prime \prime}\right)>\varepsilon|y|$. Take a function $\phi \in C_{0}^{\infty}(\boldsymbol{C})$ admitting $\phi(\tau)=1 \quad$ if $|\tau| \leqq \frac{\varepsilon}{2}$ and $\phi(\tau)=0$ if $|\tau| \geqq \varepsilon$. Then the Cauchy's integral formula (in the form of Theorem 1.2. 1 in [2]) gives

$$
\begin{aligned}
f(z)=\pi^{-n} \iint f(\zeta) \prod_{j}\left(\frac{\partial \psi\left(\xi_{j}-x_{j}+i\left(\eta_{j}-y_{j}\right) /|y|\right) / \partial \bar{\zeta}_{j}}{\zeta_{j}-z_{j}}\right) d \xi d \eta, & \\
& (\zeta=\xi+i \eta) .
\end{aligned}
$$

Applying this formula to (2.4), we have (2.5) for suitable constants $C, M$.

It remains now to prove $(d) \Rightarrow(a)$. In view of Banach-Steinhaus theorem, we have only to show the existence of the limit of $\langle f(x$ $+i y), \varphi(x)>$ as $\Gamma^{\prime} \ni y \rightarrow 0$ for each $\varphi \in C_{0}^{\infty}(\omega)$. Let $\tilde{\varphi} \in C_{0}^{1}(\tilde{U})$ be the extension of $\varphi$ into the complex domain constructed in Lemma 2. 1 .

For a fixed vector $\theta \in \Gamma^{\prime}$, we have

$$
\langle f(x+i y), \varphi(x)\rangle=\iint_{B} f(z+i y) \bar{\partial} \tilde{\varphi}(z) \wedge d z
$$

where $B=\left\{z=x+i t \theta ; \quad x \in \boldsymbol{R}^{n}, t>0\right\}$. If $f$ satisfies (2.5), (2.8) converges to the absolutely convergent integral 


$$
\iint_{B} f(z) \bar{\partial} \tilde{\varphi}(z) \wedge d z
$$

uniformly when $\Gamma^{\prime} \ni y \rightarrow 0$. This completes the proof of Theorem 2. 2 .

\section{§ 3. Wave Front Set}

We start with recalling the definition of analytic wave front set (see Hörmander [4]).

Definition 3.1. Let $f \in \mathscr{D}^{\prime}(X)$. Then the analytic wave front set $W F_{A}(f)$ of $f$ is defined as the complement, in $T^{*}(X) \backslash 0$, of the points $\left(x_{0}, \xi_{0}\right)$ such that there is an open conic neighborhood $V$ of $\xi_{0}$ and a bounded sequence $\left\{f_{N}\right\}$ in $\mathscr{E}^{\prime}(X)$ which is equal to $f$ in a common neighborhood of $x_{0}$ and satisfies the estimates

$$
\left|\hat{f}_{N}(\xi)\right| \leqq C(C N /|\xi|)^{N}, \xi \in V, N=1,2, \ldots
$$

Let $\left.W F_{A}(f)\right|_{x_{0}}=\left\{\xi \in \mathbb{R}^{n} \backslash 0 ;\left(x_{0}, \xi\right) \in W F_{A}(f)\right\}$ be the fibre over $x_{0}$. It is remarked that $\left.W F_{A}(f)\right|_{x_{0}}$ is completely characterized by the sequences of type $f_{N}=\phi_{N} f$ where $\left\{\phi_{N}\right\}$ is a bounded sequence in $C_{0}^{\infty}(X)$ which is equal to 1 in a common neighborhood of $x_{0}$ and satisfies

$$
\left|D^{\alpha+\beta} \phi_{N}\right| \leqq C_{\alpha}(C N)^{|\beta|} \text { if }|\beta| \leqq N .
$$

For the existence of such functions we refer to Lemma 2.2 in [4]. We need to extend $\phi_{N}$ into the complex domain and require more precise estimates than (2.1).

Lemma 3.2. Assume that (3.2) is valid for the sequence of functions $\phi_{N}(x) \in C_{0}^{\infty}(U)$. Then there exist the extensions $\tilde{\phi}_{2 N}(x+i y)$ $\in C_{0}^{1}(\tilde{U})$ of $\phi_{2 N}$ which satisfy the estimates

$$
\sup _{x}\left|D_{x}^{\beta} \bar{\partial} \tilde{\phi}_{2 N}(x+i y)\right| \leqq C(C|y|)^{N} N^{|\beta|} \text { if }|\beta| \leqq N .
$$

Here $C$ is indepent of $N$ and $y$. 
Proof. With the same function $\chi$ as in the proof of Lemma 2.1, we put

$$
\phi_{2 N}(x+i y)=\sum_{\alpha} \phi_{2 N}^{(\alpha)}(x)(i y)^{\alpha} \chi\left(b_{|\alpha|} y\right) / \alpha ! .
$$

Here, in this case, we make a different choice of the sequence $\left\{b_{j}\right\}$. In fact we set

$$
b_{0}=b_{1}=\cdots=b_{N}<b_{N+1}<\cdots
$$

and determine the value of $b_{0}$ so that supp $\phi_{2 N}(x) \chi\left(b_{0} y\right) \subset \tilde{U}$. Then it is easy to check

$$
\begin{aligned}
& |y|^{-N} \mid D_{x}^{\beta} \sum_{|\alpha| \leqq N}\left\{\phi_{2 N}^{\left(\alpha+1_{j}\right)}(x)(i y)^{\alpha}\left(\chi\left(b_{|\alpha|} y\right)-\chi\left(b_{|\alpha|+1} y\right)\right)\right. \\
& \left.\quad+\phi_{2 N}^{(\alpha)}(x)(i y)^{\alpha} b_{|\alpha|} \chi_{j}^{\prime}\left(b_{|\alpha|} y\right)\right\} \mid / 2 \alpha ! \\
& \quad \leqq \sup _{\alpha} C(C N)^{|\alpha|+|\beta|+1} / \alpha ! \\
& \quad \leqq C_{1}^{1+N} N^{|\beta|}, \quad|\beta| \leqq N .
\end{aligned}
$$

Taking the remaining part of the sequence, $b_{N}<b_{N+1}<\ldots$, to be increasing fast enough, we have that $\phi_{2 N} \in C_{0}^{1}(\tilde{U})$ and also that

$$
\begin{aligned}
& |y|^{-N} \mid D_{x}^{\beta} \sum_{|\alpha|>N}\left\{\phi_{2 N}^{\left(\alpha+1_{j}\right)}(x)(i y)^{\alpha}\left(\chi\left(b_{|\alpha|} y\right)-\chi\left(b_{|\alpha|+1} y\right)\right)\right. \\
& \left.\quad+\phi_{2 N}^{(\alpha)}(x)(i y)^{\alpha} b_{|\alpha|} \chi_{j}^{\prime}\left(b_{|\alpha|} y\right)\right\} / 2 \alpha ! \\
& \quad \leqq C^{1+N} N^{|\beta|}, \quad|\beta| \leqq N .
\end{aligned}
$$

This completes the proof of Lemma 3.1.

Theorem 1.2 stated in the introduction is an easy consequence of the following theorem.

Theorem 3. 3. Let $\left\{V_{\alpha}\right\}$ be a finite family of open convex proper cones in $\mathbb{R}^{n}$ and $\left\{\Gamma_{\alpha}\right\}$ a family of dual cones of $V_{\alpha}$. Then the following statesments are equivalent for any distribution $f$ defined near $x_{0} \in \mathbb{R}^{n}$.

(a) The fibre $\left.W F_{A}(f)\right|_{x_{0}}$ is contained in $\cup V_{\alpha}$.

(b) There is a neighborhood $U$ of $x_{0}$, its complex neighborhood $\tilde{U}$ and are functions $f_{\alpha} \in \mathcal{O}\left(\tilde{U} \cap T\left(\Gamma_{\alpha}^{\prime}\right)\right)$ for some open cones $\Gamma_{\alpha}^{\prime} \supseteq \Gamma_{\alpha}$ such that

$$
f=\sum_{\alpha} f_{\alpha}\left(x+i \Gamma_{\alpha}^{\prime} 0\right) \text { in } \mathscr{D}^{\prime}(U)
$$


Moreover, under the assumption (a), the decomposition (3.4) is carried out in the space of $C^{\infty}$ functions, provided that $f$ is $C^{\infty}$.

Proof. We first prove (b) $\Rightarrow$ (a). To do so it is obviously sufficient to show the following; let $g(x)=f(x+i \Gamma 0)$ for given $f \in \mathcal{O}(\tilde{U}$ $\cap T(\Gamma))$ satisfying (2.5), then $\left.W F_{A}(g)\right|_{x_{0}} \subset F$ where $F$ is the dual cone of $\Gamma$. We put $\phi_{N}=\phi_{2(N+M)}$ and $\tilde{\phi}_{N}=\tilde{\phi}_{2(N+M)}$ obtained in Lemma 3.2. Let $\theta \notin F$ and $\xi$ be in a small conical neighborhood of $\theta$ on which $\langle y, \xi\rangle\langle 0$ is valid for some $y \in \Gamma$. Since

$$
\begin{aligned}
\widehat{\xi^{\alpha}} \widehat{\psi_{N} g}(\xi) & =2 i \xi^{\alpha} \iint_{t>0} f(x+i t y)\left\langle\bar{\partial} \tilde{\phi}_{N}(x+i t y), y\right\rangle e^{-i\langle x+i t y, \xi\rangle} d x d t \\
& =2 i \sum_{\alpha_{1}+\alpha_{2}=\alpha} \frac{\alpha !}{\alpha_{1} ! \alpha_{2} !} \iint_{t>0}\left\{D_{x}^{\alpha_{1}} f(x+i t y)\right\}\left\langle D_{x}^{\alpha_{2}} \bar{\partial} \psi_{N}(x\right. \\
& +i t y), y\rangle e^{-i\langle x+i t y, \xi\rangle} d x d t,
\end{aligned}
$$

we obtain in view of (2.6) and (3.3)

$$
\begin{aligned}
\left|\xi^{\alpha} \widehat{\psi_{N} g}(\xi)\right| & \leqq \sum_{\alpha_{1}+\alpha_{2}=\alpha} \frac{\alpha !}{\alpha_{1} ! \alpha_{2} !}\left(C\left|\alpha_{1}\right|\right)^{\left|\alpha_{1}\right|} C^{1+N}(N+M)^{\left|\alpha_{2}\right|} \\
& \leqq C_{1}\left(C_{1} N\right)^{N}, \quad|\alpha| \leqq N .
\end{aligned}
$$

This implies $\left.\theta \notin W F_{A}(f)\right|_{x_{0}}$.

For the converse verification, it should be noted that one may assume $x_{0}=0$ and $f \in C_{0}^{\infty}$. In fact let $W$ be an open cone such that

$$
\left.\left(\left.W F(f)\right|_{0} \subset\right) W F_{A}(f)\right|_{0} \Subset W \Subset \bigcup_{\alpha} V_{\alpha}
$$

and set with a function $\psi$ having small support and equal to 1 near 0 ,

$$
\begin{aligned}
g(x) & =(2 \pi)^{-n} \int_{W^{c}} e^{i<x, \xi>} \widehat{\psi f}(\xi) d \xi \\
& =\phi f(x)-(2 \pi)^{-n} \int_{W} e^{i<x, \xi>} \widehat{\phi f}(\xi) d \xi,
\end{aligned}
$$

where we take the last integration in the distribution sense. This integral can be written in a sum of boundary values of holomorphic functions from the directions of dual cones of $W \cap V_{\alpha}$. By the implication (b) $\Rightarrow$ (a) just proved (or rather by a direct proof) the second equality implies $\left.W F_{A}(g)\right|_{0} \subset \bar{W}$. On the other hand the first 
implies $g(x) \in C^{\infty}$ for one may assume supp $\phi$ is so small that $\widehat{\phi f}(\xi)$ is rapidly decreasing on $W^{c}$. If $g$ has a corresponding decomposition as in (3.4), then $f(=\phi f$ near 0$)$ has too. Thus our claim is justified.

One can now take a bounded sequence $\left\{f_{N}\right\}$ in $C_{0}^{\infty}$ which satisfies (3.1) on $W^{\circ}$ ( $W$ introduced in (3.5)) and $f_{N}=f$ in the region $\left\{x ;|x|^{2}<a\right\}$ for small $\mathrm{a}>0$. We shall consider the Fourier transform of $f(x) \exp \left(-\xi_{0}|x|^{2}\right)$ with additional dual parameter $\xi_{0}$. This idea is due to Bros-Iagornitzer [1]. We have

$$
\begin{aligned}
& \left|\int f(x) \exp \left(-\xi_{0}|x|^{2}-i\langle x, \xi\rangle\right) d x\right| \\
\leqq & \left|\int\left(f-f_{N}\right) \exp \left(-\xi_{0}|x|^{2}-i\langle x, \xi\rangle\right) d x\right| \\
+ & \left|\int f_{N} \exp \left(-\xi_{0}|x|^{2}-i\langle x, \xi\rangle\right) d x\right|,
\end{aligned}
$$

where $\xi_{0}$ varies in the interval $[1, \infty)$. The first term of the right hand side of (3.7) is bounded by $C_{j}(1+|\xi|)^{-j} \xi_{0}^{j} \mathrm{e}^{-\xi_{0} a}$ for the sequence $\left\{f-f_{N}\right\}$ is bounded in $C_{0}^{\infty}$ and has support in $\left\{x ;|x|^{2} \geqq a\right\}$. Since the Fourier transform of $\exp \left(-\xi_{0}|x|^{2}\right)$ is equal to $\left(\pi / \xi_{0}\right)^{n / 2} \exp \left(-|\eta|^{2}\right.$ $\left./ 4 \xi_{0}\right)$, it follows

$$
\begin{aligned}
& \left|\int f_{N}(x) \exp \left(-\xi_{0}|x|^{2}-i\langle x, \xi\rangle\right) d x\right| \\
& \quad=(2 \pi)^{-n}\left|\int \hat{f}_{N}(\eta)\left(\pi / \xi_{0}\right)^{n / 2} \exp \left(-|\xi-\eta|^{2} / 4 \xi_{0}\right) d \eta\right| .
\end{aligned}
$$

If $\xi \in F=\left(\bigcup_{\alpha} V_{\alpha}\right)^{c}$ and $\eta \in W$ we have $|\xi-\eta| \geqq c(|\xi|+|\eta|)$ for some $c>0$. On the other hand when $|\xi-\eta|<\frac{1}{3}(|\xi|+|\eta|)$ we have $\frac{1}{2}|\xi|<|\eta|$. Hence we can estimate (3.8) by

$$
C\left((C N /|\xi|)^{N}+\exp \left(-\delta|\xi|^{2} / \xi_{0}\right)\right), \quad \xi \in F .
$$

Furthermore it is easy to see

$$
\inf _{N}(C N /|\xi|)^{N} \leqq C^{\prime} e^{-\tilde{o}^{\prime}|\xi|} .
$$

Summing up the estimates obtained, we have with other constants $b>0$ and $C_{j}$,

$$
\left|\int f(x) \exp \left(-\xi_{0}|x|^{2}-i\langle x, \xi\rangle\right) d x\right|
$$




$$
\leqq C_{j}(1+|\xi|)^{-j}\left(\exp \left(-b \xi_{0}\right)+\exp (-b|\xi|)\right), j=1,2, \ldots,
$$

when $\xi \in F$ and $1 \leqq \xi_{0} \leqq|\xi|$.

Keeping this estimate in mind, we shall consider a kind of inverse Fourier transform. We define the $n$-form

$$
\begin{aligned}
& W(f)=(2 \pi)^{-n} \exp \left(\xi_{0}|x|^{2}+i\langle x, \xi\rangle\right) \sum_{k=0}^{n}(-1)^{k} W_{k}(f)\left(\xi_{0}, \xi, x\right) \\
& d \xi_{0} \wedge \ldots \wedge d \xi_{k-1} \wedge d \xi_{k+1} \ldots \wedge d \xi_{n},
\end{aligned}
$$

where

$$
\begin{aligned}
& W_{0}(f)=\int f(y) \exp \left(-\xi_{0}|y|^{2}-i\langle y, \xi\rangle\right) d y, \\
& W_{k}(f)=\int f(y) \exp \left(-\xi_{0}|y|^{2}-i\langle y, \xi\rangle\right) \rho_{k}(y, x) d y .
\end{aligned}
$$

We set $\rho_{k}(y, x)=i\left(x_{k}+y_{k}\right)$ so that

$$
\begin{aligned}
& d_{\xi_{0, \xi}} W(f)=(2 \pi)^{-n} \exp \left(\xi_{0}|x|^{2}+i\langle x, \xi\rangle\right) \int f(y) \\
& \quad \times \exp \left(-\xi_{0}|y|^{2}-i\langle y, \xi\rangle\right)\left(|x|^{2}-|y|^{2}\right. \\
& \left.\quad+i \sum\left(x_{k}-y_{k}\right) \rho_{k}\right) d y d \xi_{0} \wedge \ldots \wedge d \xi_{n}=0 .
\end{aligned}
$$

Since $\rho_{k}(y, x)$ with $1 \leqq k \leqq n$ and $|x|^{2}<b$ are uniformly bounded holomorphic functions on every bounded set in $\boldsymbol{C}_{y}^{n}$, one may assume that $W_{k}$ also satisfy (3.9) with the same constants when $|x|^{2}<b$.

The Fourier inversion formula gives

$$
f(x)=\int_{\xi_{0}=1} W(f) .
$$

One can write when $|x|^{2}<b$

$$
\int_{\xi_{0}=1, \xi \in \cup_{\alpha} V_{\alpha}} W(f)=\sum_{\alpha} h_{\alpha}\left(x+i \stackrel{\circ}{\Gamma}_{\alpha} 0\right)
$$

with $C^{\infty}$ functions $h_{\alpha}\left(x+i \dot{\Gamma}_{\alpha} 0\right)$ which are the boundary values of holomorphic functions $h_{\alpha}$ from the directions $\dot{\Gamma}_{\alpha}$ the interior of $\Gamma_{\alpha}$.

The remaining part of the integral domain of (3.11) can be distorted as

$$
\int_{\xi_{0}=1, \xi \in F} W(f)=\int_{1 \leqq \xi_{0} \leqq|\xi|, \xi \in \partial F} W(f)+\int_{\xi_{0}=|\xi|, \xi \in F} W(f)
$$

if $x$ is small. In fact, since $W(f)$ is closed, the difference between 
the both sides of (3.12) is the limit as $R \rightarrow \infty$ of the integral of $W(f)$ on the domain $1 \leqq \xi_{0} \leqq|\xi|=R, \xi \in F$. This integral must have the bound

$$
C_{j}(1+R)^{-j} \int_{1}^{R} \exp \left(\xi_{0}\left(|x|^{2}-b\right)\right) d \xi_{0}
$$

which tends to 0 as $R \rightarrow \infty$ and $|x|^{2}<b$.

Now the first term in the right hand side of (3.12) is written in a sum of $C^{\infty}$ functions which are the boundary values of holomorphic functions from the $\dot{\Gamma}_{\alpha}$ directions in the above sense when $|x|^{2}<b$. It is also easy to see that (3.9) implies the second term is real analytic when $|x|^{2}<b$. Hence we have a desired decomposition of $f$ from the directions $\dot{\Gamma}_{\alpha}$. To obtain a decomposition in terms of the cones $\Gamma_{\alpha}^{\prime} \rightrightarrows \Gamma_{\alpha}$, we have only to shrink $V_{\alpha}$ suitably in the above argument. Thus the proof of Theorem 3.3 is completed.

Finally we give a characterization of $C^{\infty}$ wave front set, which is an analogue of Theorem 1.2.

Theorem 3.4. Let $f \in \mathscr{D}^{\prime}(X) \quad$ and $\left(x_{0}, \xi_{0}\right) \in T^{*}(X) \backslash 0$. Then $\left(x_{0}, \xi_{0}\right) \notin W F(f)$ if and only if there exists a finite family $\left\{\Gamma_{\alpha}\right\}$ of open convex cones in $\mathbb{R}^{n}$ and a complex neighborhood $\tilde{U}$ of $x_{0}$ such that one can write

$$
f=\sum_{\alpha} f_{\alpha}\left(x+i \Gamma_{\alpha} 0\right)
$$

in a neighborhood of $x_{0}$ with such $f_{\alpha} \in \mathcal{O}\left(\tilde{U} \cap T\left(\Gamma_{\alpha}\right)\right)$ that $f_{\alpha}\left(x+i \Gamma_{\alpha} 0\right)$ $\in C^{\infty}$ near $x_{0}$ for every $\alpha$ satisfying $\Gamma_{\alpha} \subset\left\{y ;\left\langle y, \xi_{0}\right\rangle \geqq 0\right\}$.

Proof. Suppose that (3.13) is valid for such cones $\left\{\Gamma_{\alpha}\right\}$. Taking the subfamily $\{\beta\} \subset\{\alpha\}$ of indices defined by $\Gamma_{\beta} \cap\left\{y ;\left\langle y, \xi_{0}\right\rangle\right.$ $\langle 0\} \neq \phi$, we see by Theorem 1.2 that $\left(x_{0}, \xi_{0}\right) \notin W F_{A}\left(\sum_{\beta} f_{\beta}\left(x+i \Gamma_{\beta} 0\right)\right)$ and then that $\left(x_{0}, \xi_{0}\right) \notin W F(f)$.

Conversely assume that $\left.W F(f)\right|_{x} \subset \bigcup_{\alpha} V_{\alpha}$ in an open neighborhood $U$ of $x_{0}$ for a finite family $\left\{V_{\alpha}\right\}$ of open convex proper cones such that $\xi_{0} \notin \cup_{\alpha} V_{\alpha}$. Then choosing a function $\chi \in C_{0}^{\infty}$ having support in $U$ and equal to 1 near $x_{0}$, we have close to $x_{0}$ 


$$
\begin{aligned}
f(x) & =(2 \pi)^{-n} \int_{F} \widehat{\chi f}(\xi) e^{i<x, \xi>} d \xi \\
& +(2 \pi)^{-n} \int_{\bigcup_{\alpha} V_{\alpha}} \widehat{\chi f}(\xi) e^{i<x, \xi>} d \xi
\end{aligned}
$$

where $F=\left(\cup_{\alpha} V_{\alpha}\right)^{c}$.

The first term of the right hand side is indeed $C^{\infty}$ and written in a sum of $C^{\infty}$ boundary values of holomorphic functions from some directions. The second term is decomposed into a sum of boundary values of holomorphic functions from $\dot{\Gamma}_{\alpha}^{\circ}$ directions. Here since $\xi_{0} \notin V_{\alpha}$ the open dual cone $\dot{\Gamma}_{\alpha}$ meets the set $\left\{y ;\left\langle y, \xi_{0}\right\rangle\langle 0\}\right.$, which completes the proof of the theorem.

\section{References}

[1] Bros, $J$. and Iagornitzer, D., Causality and local analyticity: mathematical study, Ann. Inst. Henri Poincare, 18, No. 2 (1973), 147-184.

[2] Hörmander, L., An introduction to complex analysis in several variables, D. van Nostrand. Publ. Co., Princeton, N. J. 1966.

[3] Hörmander, L,. On the singularities of solutions of partial differential equations, Comm. Pure Appl. Math., 23 (1970), 329-358.

[4] Hörmander, L., Uniqueness theorems and wave front sets for solutions of linear differential equations with analytic coefficients, Comm. Pure Appl. Math., 24 (1971), 671-704.

[5] Martineau, A., Distributions et valeurs au bord des fonctions holomorphes, Proc. Intern. Summer Course on the theory of Distributions, Lisbon, (1964), 195-326.

[6] Nishiwada, K., On the local surjectivity of analytic partial differential operators in the space of distributions with given wave front sets, Surikaiseki-kenkyusho kokyuroku, R. I. M. S., Kyoto Univ., 239 (1975), 19-30.

[7] Sato, M., Theory of hyperfunctions I, II, J. Fac. Sci. Univ. Tokyo, 8 (1960), 139-193, 387-436.

[8] Sato, M., Regularity of hyperfunction solutions of partial differential equations, Actes Congres Intern. Math., (1970) Tome 2, 785-794. 